\section{PWE-037 PROTON PUMP INHIBITORS AND HYPOMAGNESAEMIA}

doi:10.1136/gutjnl-2012-302514d.37

S Hebbar, ${ }^{*}$ N Sharma, P Wilson. Department of Gastroenterology, Heartlands Hospital, Birmingham, UK

Introduction Hypomagnesaemia is a rare and potentially serious complication of PPI therapy. About 30 cases of severe hypomagnesaemia have been reported in long term PPI users. ${ }^{1}$

Methods We describe two cases recently admitted to our hospital with symptomatic hypomagnesaemia and a history of long term PPI usage.

Results Patient 1-A 54-year-old female was admitted with nausea, vomiting and abdominal discomfort for few months. Patient was on lansoprazole for more than a year. On admission, magnesium level was $0.42 \mathrm{mmol} / 1$. Patient was treated with magnesium supplements and was sent home with increased dose of lansoprazole. Over the next few weeks, magnesium level dropped to $0.4 \mathrm{mmol} / \mathrm{l}$ in spite of supplementation. On stopping PPI, magnesium returned to normal levels in 4 weeks time. Patient 2-A 75-year-old female was admitted with vomiting and lethargy. Patient was on PPI for more than 18 years. Magnesium level was $0.13 \mathrm{mmol} / \mathrm{l}$ and calcium $1.62 \mathrm{mmol} / \mathrm{l}$. Patient was treated with intravenous supplements and discharged on oral magnesium. Within 2 months, patient was readmitted with similar complaints and magnesium level was again noted to be $0.13 \mathrm{mmol} / \mathrm{l}$. OGD and CT abdomen were normal. Urinary magnesium levels were normal. Magnesium levels did not normalise in spite of supplementation. Omeprazole was stopped and magnesium started improving over the next 4 weeks.

Conclusion Hypomagnesaemia is a under recognised complication of long term PPI therapy, which responds rapidly on stopping PPI. In few of the reported cases, a causal relationship with PPI use has been established by recurrence of hypomagnesaemia after rechallenge. ${ }^{2}$ The cause of hypomagnesaemia is poorly understood. Mutation of TRPM 6/7 gene which is involved in the active transcellular pathway of intestinal absorption of magnesium is one of the postulated mechanism. ${ }^{2}$ Monitoring magnesium levels in patients on long term PPI should be considered. ${ }^{3}$ This rare complication might not even be so uncommon as more people become aware of the association.

Competing interests None declared

\section{REFERENCES}

1. Cundy T, Mackay J. Proton pump inhibitors and severe hypomagnesaemia. Curr Opin Gastroenterol 2011;27:180-5.

2. Negri AL, Valle EE. Hypomagnesaemia/hypokalemia associated with the use of esomeprazole. Curr Drug saf 2011:6:204-6.

3. FDA Drug Safety Communication. Low Magnesium Levels can be Associated with Long-Term use of Proton Pump Inhibitor drugs (PPIs).

\section{PWE-038 INFLUENCE OF POSTOPERATIVE MORBIDITY ON LONGTERM CANCER SURVIVAL AFTER OESOPHAGOGASTRIC SURGERY}

doi:10.1136/gutjnl-2012-302514d.38

A Eltweri, S Satheesan, ${ }^{*}$ S Ubhi, D Bowrey. Department of Surgery, University Hospitals of Leicester NHS Trust, Leicester, UK

Introduction Previous studies have shown that postoperative adverse events after colorectal resection predict a poor prognosis with early cancer relapse. The aim of this study was to report the outcome of patients undergoing oesophagogastric resection to assess the influence of in-hospital factors on longterm cancer survival.

Methods Retrospective review of patients undergoing oesophagogastric resection for carcinoma during the years 2006-2010 at our institution. Minimum follow-up of 12 months was required.
Results The study population was 164 patients (110 male) of median age 64 years (range 32-84). 84 underwent oesophagectomy, 80 gastrectomy. 99 received neoadjuvant chemotherapy. The 90 -day, 1-year and 3-year survivals were 92\%, 84\% and 49\% respectively. 69 patients (42\%) developed postoperative complications (commonest: pneumonia $19 \%$, anastomotic leak $7 \%$, wound infection $6 \%$ ). None of tumour site (oesophagus vs stomach, $\mathrm{p}=0.73$ ), length of ITU stay ( $<3$ days vs $>3$ days, $p=0.50$ ) or development of postoperative complications $(p=0.70)$ influenced longterm prognosis. The only two factors that influenced longterm outcome were UICC stage $(p<0.001)$ and circumferential resection margin (positive vs negative, $\mathrm{p}<0.001)$.

Conclusion Patients experiencing postoperative morbidity can expect the same longterm oncologic outcome as those not suffering these early setbacks.

Competing interests None declared.

\section{PWE-039 ROUTINE WATER SOLUBLE CONTRAST SWALLOW HAS LIMITED CLINICAL VALUE IN THE DETECTION OF ANASTOMOTIC LEAKS FOLLOWING OESOPHAGECTOMY}

doi:10.1136/gutjnl-2012-302514d.39

S Love, ${ }^{*}$ S Bruce, T S Athwal, N Howes, M Hartley. Upper Gl Surgery, Liverpool Heart and Chest Hospital NHS Foundation Trust, Liverpool, UK

Introduction Water-soluble contrast swallow (WSCS) is performed following oesophagectomy to assess anastomotic integrity before commencing oral intake. This study, the largest in the UK to date, challenges the routine use of WSCS following oesophagectomy.

Methods All patients undergoing open transthoracic oesophagectomy for oesophageal cancer with intrathoracic anastomosis, within a supra-regional upper GI cancer centre, were registered on a prospective database between 2006 and 2011. WSCS results, anastomotic leak rate and the modality of leak detection were analysed.

Results During the study period, 116 oesophagectomies were performed. WSCS was undertaken in 97 (84\%) cases on a median of day 5 (range 3-8) post operatively; 95 (98\%) WSCS reported no evidence of a leak, two studies reported a leak and one study was equivocal. WSCS was not performed in 19 (16\%) cases; 10 patients developed early clinical signs suggestive of an anastomotic leak and were immediately imaged by CT, eight had a prolonged ITU stay due to cardiorespiratory complications while one patient died perioperatively. There were $6(5 \%)$ anastomotic leaks of which three patients had immediate CT due to clinical deterioration while three patients had routine WSCS. WSCS was followed by CT in two patients due to a positive or equivocal finding. One patient had no WSCS evidence of a leak but then developed sepsis and CT confirmed a leak. Clinical signs suggestive of a leak were evident in all patients within 7 days post-operatively.

Conclusion Routine WSCS has limited value in the detection of anastomotic leak following oesophagectomy. All patients with an anastomotic leak developed significant clinical signs of a leak that were subsequently confirmed by CT imaging. WSCS altered the management in just $2 \%$ of cases.

Competing interests None declared.

\section{PWE-040 LYMPH NODE METASTASIS IN EARLY OESOPHAGEAL CANCER: EXPERIENCE FROM A 10 YEAR UK COHORT}

doi:10.1136/gutjnl-2012-302514d.40

${ }^{1} \mathrm{~T}$ S Athwal, ${ }^{* 1} \mathrm{~S}$ Love, ${ }^{1} \mathrm{~S}$ Bruce, ${ }^{2} \mathrm{R}$ Page, ${ }^{1} \mathrm{M}$ Hartley, ${ }^{1} \mathrm{~N}$ Howes. ${ }^{1}$ Upper G/ Surgery, Liverpool Heart and Chest Hospital NHS Foundation Trust, Liverpool, UK; ${ }^{2}$ Thoracic \& 
Upper Gl Surgery, Liverpool Heart and Chest Hospital NHS Foundation Trust, Liverpool, UK

Introduction Surgical resection is considered the gold standard curative treatment for oesophageal cancer, however, there is debate regarding the role of endoscopic resection for "early" (Tis, T1) oesophageal cancer. However, studies have identified that a significant number of early cancers will have lymph node metastases (LNM) at time of diagnosis. The aim of this study was to determine the incidence of LNM in early oesophageal cancer patients in a 10 year UK cohort.

Methods A total of 482 consecutive patients who had transthoracic oesophagectomy for adenocarcinoma or squamous cell carcinoma, at a supra-regional upper GI cancer centre, were registered on a prospective database between 2002 and 2012. Patients with early oesophageal cancer were retrieved from the database based on pre-operative endoscopic and radiological staging.

Results Of the 482 patients, $53(11 \%)$ had early oesophageal cancer, of which $41(77 \%)$ were adenocarcinoma, 11 (21\%) squamous cell carcinoma and $1(2 \%)$ carcinoma in situ. The median lymph node harvest was 14 (IOR 9-17). Lymph node metastases were present in $4(7.6 \%)$ cases, all of which had tumour extending into the submucosal layer.

Conclusion Early oesophageal cancer has a significant risk of lymph node involvement especially when the submucosal layer is breached by tumour. In our opinion, surgical resection remains the gold standard to achieve cure in patients with early cancer.

Competing interests None declared.

\section{PWE-041 OBESITY IS ASSOCIATED WITH IMPROVED SURVIVAL IN SURGICALLY RESECTED OESOPHAGEAL CANCER}

\section{doi:10.1136/gutjnl-2012-302514d.41}

${ }^{1} \mathrm{~T}$ S Athwal, ${ }^{* 1} \mathrm{~S}$ Love, ${ }^{1} \mathrm{~S}$ Bruce, ${ }^{2} \mathrm{R}$ Page, ${ }^{1} \mathrm{M}$ Hartley, ${ }^{1} \mathrm{~N}$ Howes. ${ }^{1}$ Upper Gl Surgery, Liverpool Heart and Chest Hospital NHS Foundation Trust, Liverpool, UK; ${ }^{2}$ Thoracic \& Upper GI Surgery, Liverpool Heart and Chest Hospital NHS Foundation Trust, Liverpool, UK

Introduction Obesity is an increasing health burden in the western world and a known risk factor for oesophageal cancer. The aim of this study was to determine the impact of obesity on circumferential resection margin (CRM), lymph node yield (LNY) and survival among a 10 year cohort of surgically resected oesophageal cancer patients.

Methods All patients who underwent transthoracic oesophagectomy for histologically proven adenocarcinoma or squamous cell carcinoma, performed at a supra-regional upper GI cancer centre, were registered on a prospective database between 2002 and 2012. Total LNY, CRM and survival was compared between different BMI groups: normal (18.5-24.9), overweight (25-30), and obese $(>30)$. Patients who were malnourished prior to surgery were excluded from the analysis.

Results A total of 482 consecutive patients underwent oesphagectomy during the study period, of which the complete dataset was available for 441 (91\%); normal (155), overweight (177) and obese (91). The median lymph node yield was 16 (IOR 11-22), normal 15 (10-23), overweight $17(12-23)$ and obese 15 (10-21), with no significant difference seen between the three groups $\left(p=0.4565, \chi^{2}\right.$ test). The CRM was involved in $30(27 \%)$ of the obese patients compared to 55 (31\%), 54 (35\%) and of overweight, healthy patients respectively ( $p=0.0615, \chi^{2}$ test).

Conclusion Obesity appears to confer a survival advantage in patients undergoing surgical resection for oesophageal cancer. Interestingly, there appeared to be a trend towards an uninvolved CRM in obese patients but no significant impact on the lymph node yield.

Competing interests None declared.

\section{PWE-042 THE RELEVANCE OF THE SIEWERT CLASSIFICATION IN THE ERA OF MULTIMODAL THERAPY FOR ADENOCARCINOMA OF THE GASTRO-OESOPHAGEAL JUNCTION}

doi:10.1136/gutjnl-2012-302514d.42

1,2 F Noble, ${ }^{2}$ S B Bailey, ${ }^{2} \mathrm{~J} J$ Kelly, ${ }^{2} \mathrm{~J}$ P Byrne, ${ }^{1,2} \mathrm{~T}$ J Underwood. ${ }^{1}$ Cancer Sciences Unit, Faculty of Medicine, University of Southampton, Southampton, UK; ${ }^{2}$ Department of Surgery, University Hospital Southampton Foundation Trust, Southampton, UK

Introduction Since the early 1980s the Siewert classification has been used to plan treatment for tumours of the gastro-oesophageal junction. However, the relationship between tumour site and survival has not been conclusively demonstrated, with conflicting outcomes in the largest series, before the widespread application of neoadjuvant chemotherapy. The aim of this study was to evaluate whether there were differences in the biology and clinical characteristics of adenocarcinomas by Siewert type, in a contemporary cohort of patients, in whom the majority had received neoadjuvant chemotherapy. The relationship of the surgical approach and tumour site with patient survival was also assessed.

Methods A prospective database was reviewed for all patients who underwent resection for adenocarcinoma of the distal oesophagus and gastro-oesophageal junction from 2005 to 2011. In our unit, based on pre-operative assessment, distal oesophageal, type I and II tumours are treated as oesophageal cancer, with transthoracic procedures. Type III tumours are treated as gastric cancer with an abdominal approach. Classification systems used for analysis included TNM 7 for staging, Clavien-Dindo for grading complications and Siewert with final tumour site determined from the pathological specimen. Survival was estimated by Kaplan-Meier analysis excluding inpatient deaths $(n=4)$ and $R 1$ resections $(n=42)$. Results 216 patients underwent oesophagogastric resection: 133 for type I, 51 for type II and 33 for type III tumours. Median follow-up was 2.94 years. $62.5 \%$ of patients received neoadjuvant chemotherapy with no difference between groups. There were no significant differences in age, sex, $\mathrm{pT}$ stage, $\mathrm{pN}$ stage, $\mathrm{pM}$ stage, ASA, or inpatient complications between patients with adenocarcinoma based on their Siewert classification. Type I tumours were significantly associated with coexisting Barrett's metaplasia (presence of Barrett's: Type I 58.3\%, Type II 21.6\%, Type III 9.1\%; pType II > Type I). Median overall survival was significantly shorter for more distal tumours (Type I: 4.96 years vs Type II: 3.3 years vs Type III: 2.64 years; $p=0.04)$. The surgical approach did not influence survival for all tumour types and had no impact on the rate or severity of complications.

Conclusion This study demonstrates significant differences in the biological characteristics of adenocarcinomas of the gastro-oesophageal junction based on their anatomical topographical sub-classification. In the era of multimodal therapy overall survival is worse for tumours arising at or below the gastro-oesophageal junction compared with oesophageal tumours.

Competing interests None declared.

\section{PWE-043 CHANGING EPIDEMIOLOGY OF FOOD BOLUS IMPACTION IS EOSINOPHILIC OESOPHAGITIS TO BLAME?}

doi:10.1136/gutjnl-2012-302514d.43

V Mahesh, ${ }^{*}$ R Holloway, $Q$ N Nguyen. Department of Gastroenterology, Royal Adelaide Hospital, Adelaide, Australia

Introduction The epidemiology of acute food bolus impaction (FBI) of the oesophagus in adults remains unclear. The incidence of eosinophilic oesophagitis (EE) is increasing and is a well-known cause for FBI. Currently, there are no data on the epidemiological changes in FBI and its relationship to EE. 